\title{
The effect of product variety on purchase probability
}

\author{
Mark Heitmann · Andreas Herrmann · Christian Kaiser
}

Received: 16 November 2006/Revised: 21 February 2007 / Accepted: 7 March 2007/

Published online: 8 May 2007

(C) Springer-Verlag 2007

\begin{abstract}
Regarding the effect of product variety on purchase probability, there exist findings which demonstrate a positive effect of variety for small assortments and a negative effect of variety for large assortments. Despite these results, little evidence exists about the causal mechanism of this effect. We conduct a field study among German consumer electronics customers to investigate the previously proposed constructs of anticipated product utility, anticipated regret and evaluation costs. The results suggest that anticipated regret and evaluation costs play a powerful role in explaining the negative link between variety and purchase probability for high variety assortments. Anticipated product utility on the other hand serves to explain part of the positive causality for low variety assortments. The results obtained give rise to recommendations for the planning of assortments.
\end{abstract}

Keywords Product variety · Mediator analysis · Regret · Evaluation costs · Product utility

\section{JEL Classification Numbers $\mathrm{M} 30 \cdot \mathrm{M} 31$}

\section{Why variety matters}

Recent research has emphasized the importance of product variety and how it impacts profitability and specifically consumers decisions on whether or not to purchase a product (e.g., Iyengar and Lepper 2000; Gourville and Soman 2005).

\footnotetext{
M. Heitmann · A. Herrmann $(\bowtie)$

Research Institute for Business Metrics and Audi Lab for Market Research, University of St Gallen, Gallen, Switzerland

e-mail: andreas.herrmann@unisg.ch

C. Kaiser

Risk Measurement und Management, Credit Suisse Zürich, Zurich, Switzerland
} 
This work has shown that variety can have a substantial impact on revenues. For example, Boatwright and Nunes (2001) present field data where changes in variety resulted in increased revenues of up to $11 \%$.

Given the heterogeneity in tastes across consumers, economic theory would predict that larger variety assortments should be beneficial to consumers and consequently result in increased sales (e.g., Kreps 1979). Intuitively, this assumption seems plausible. At the very least it seems reasonable that people cannot have too many options. For example, if a consumer is looking for a DVD player and is trying to decide between three models, then an additional fourth model should not decrease her purchase intention. After all, the fourth option may be the exact option she is looking for. If this proves not to be the case, she can return to the original three options previously compared. Consequently, consumers should not be worse off with additional options but should instead benefit from larger variety assortments. Certain empirical evidence seems to support this claim. Positive relationships between variety and assortment size have been shown for satisfaction and confidence in the impeding decision (Jacoby et al. 1974), the attractiveness of an assortment (Ratner et al. 1999) and choice of an assortment from which to shop from (Kahn and Lehmann 1991; Glazer et al. 1991; Broniarczyk et al. 1998).

However, recent research calls this positive belief about variety into question. Specifically, research demonstrating this relation has been criticised for failing to provide a close approximation of reality since only small- to medium-range assortments have been taken into consideration. For example, Kahn and Lehmann (1991) present their subjects with only two (small variety) and six (large variety) options. Also, Jacoby et al. (1974) limit themselves to 4, 8 and 12 options. Product variety that exceeds such amounts may well break the boundaries of the span of perceptual dimensionality (Miller 1956). Several studies have demonstrated that key marketing variables such as confidence in the purchase decision, satisfaction, and also the decision to transact a purchase are negatively influenced, given a sufficiently large number of alternatives (Malhotra 1982; Huffman and Kahn 1998; Schwartz et al. 2002). Results obtained by Iyengar and Lepper (2000) are particularly striking. Employing a field experiment, the authors show that the purchaser share of customers interested in marmalade falls from 30 to $3 \%$ if the number of options is increased from 6 to 24 .

Thus, current empirical evidence clearly shows an inverted U-shaped relationship between perceived variety and purchase likelihood of consumers planning a purchase. Specifically, up to an optimal point, perceived variety appears to increase purchase probability, after which, purchase probability decreases. Despite the results supporting this notion, previous research has been largely limited to the demonstration of effects. Several intervening variables that might explain the causal mechanisms behind the phenomenon have been merely proposed but remain empirically untested. For instance, Iyengar and Lepper (2000, 1003) conclude by asking "how can there be so much dissatisfaction in the face of so much opportunity?', and call for further research on intervening variables. Other authors are more specific and call for research on potential mediators, such as anticipated regret (Schwartz et al. 2002), evaluation costs (Levav et al. 2006) or the 
attractiveness, i.e., anticipated utility, of the most favourable option (Chernev 2006). Knowledge about such mediators is important as it highlights levers companies may use to increase or decrease the effect of variety.

This paper aims to fill this gap by theoretically exploring why and under which conditions these variables might explain the relationship between variety and purchase probability. In addition, empirical results of a field study are presented that tests the explanatory power of these factors and demonstrates how they are related. With the aid of these results, this article aims to illuminate the causal process between variety and purchase probability.

\section{Hypotheses on the causal mechanism between variety and purchase probability}

Given the inverted U-shaped relation between variety and purchase probability found in previous research, different causal mechanisms may be at work for low and high variety assortments. Consequently, the first question to be considered is why perceived variety increases the probability that consumers will make a purchase if the assortment is below a medium range. In answering this question, the important concept of utility comes to the fore. Economists traditionally assume that individuals will choose the product that maximizes their subjective utility (Hicks 1939). Kahneman et al. (1997) explain that one must distinguish between the anticipated utility relevant to the decision and the utility actually experienced by individuals. It is assumed that individuals will choose the option through which the expected need fulfillment will be maximized (Vodopivec 1992; Harsanyi 1997). In reality, it is often not possible for individuals to identify and compare all available options because the amount of information is impossible either to obtain or to process. Consequently, there exist search costs in the form of limited cognitive and monetary resources (Shugan 1980; Stigler 1961). This decision of whether to choose one of the presently available alternatives-as shown in numerous empirical studies-involves consumers weighing anticipated utility against the cost of a continued search (Ratchford 1982; Corbin 1980). Accordingly, the "no-choice" option is chosen when none of the available alternatives appears to be sufficiently attractive or when the decision-maker suspects that she can identify alternatives with a higher anticipated utility by means of a continued search (Dhar 1997).

Larger assortments allow consumers to satisfy individual needs by permitting better compatibility between individual utility functions and the characteristics of the alternatives offered (Chernev 2003; Lancaster 1990; Loewenstein 1999). Larger assortments thus increase the probability that the anticipated utility of a presently available option will exceed the utility of a continued search. Accordingly, individuals who are hesitant to make a purchase indicate the expected utility of the best alternative as one of the central reasons for their decision to purchase a product anyway (Greenleaf and Lehmann 1995). Other research confirms that individuals explicitly cite the anticipated utility of the best alternative as the reason for their preference for large selections over small ones (Broniarczyk et al. 1998; Kahn et al. 
1987). According to economic theory, however, it cannot be expected that new options will create constant additional utility. More reasonable appears the assumption of a decreasing marginal utility due to additional options.

In summary, it is thus to be expected that the relation between product variety and purchase probability can be explained by the anticipated utility of the best alternative. If a decreasing marginal utility is indeed present, anticipated utility should function as a mediator, especially with small assortments.

H1: with small assortments, the positive effect of variety on purchase probability is mediated by the anticipated utility of preferred alternatives.

If anticipated utility is able to explain the relation between variety and purchase probability for small assortments, then the question arises as to what explanation can be found for the negative correlation with large assortments. An obvious construct is decision costs, which increase with the number of alternatives. Social psychologists have long known that individuals can be conceptualized as cognitively lazy (Zipf 1949). They are prepared to invest mental resources in a task such as decision-making only when they anticipate sufficient compensation or when they feel compelled to do so. In order to keep the decision costs at a minimum, so argue decision theorists, individuals invest exactly that level of effort in a purchase decision as will allow them to exceed at least a minimum level of expected satisfaction (Simon 1955, 1990; March 1978).

Customers can pursue any number of paths to reach that goal. Thus, a variety of information processing strategies is conceivable with which individuals can reach a satisfactory information level (Frisch and Clemen 1994; Beach and Mitchell 1978; Shugan 1980). Individuals have at their disposal a process of the highest possible precision by which they objectively process and subjectively assess all units of information and condense them into a preferential judgment. To be able to cope with more complex problems, they can also access a number of heuristics. Heuristic decision-makers save on cognitive effort by ignoring information and in this way risk making a sub-optimal selection. Decision theorists speak of a lower precision in relation to complete information processing (Anderson 2003; Bettman et al. 1998).

A number of empirical studies confirm the most well-known model by Payne et al. (1993), according to which individuals try to act in such a way so as not to exceed an individually varying maximum of effort and not to fall short of a minimum of precision (Timmermans 1993; Johnson and Meyer 1984; Ford et al. 1989). Within those two parameters, individuals choose the action which is most closely aligned with their individual preferences between precision and effort. Medium variety can already lead to a situation in which complete information processing is not feasible since the number of options at that level gives rise to a disproportionate increase in the decisional effort required. If variety continues to increase, also preferred heuristics with reduced information processing increasingly exceed the maximum cost of decision making until finally, from a certain level of variety on, the satisfaction minimum between precision and effort is achieved with no further course of action. Independent of where the exact parameters of minimum precision and of maximum cost are for individuals, it is evident that with a large product variety fewer and fewer heuristics are available. 
In such a constellation, the decision-maker is in conflict between two unwanted states: producing an unpleasantly high level of effort in product choice or transacting a purchase at an unappealingly low level of precision. Such conflicts especially reduce the motivation of making a choice and consequently purchasing a product (Anderson 2003; Tversky and Shafir 1992). As noted, consumers generally possess a number of strategies to deal efficiently with low- to medium-range product variety (Payne et al. 1993). This suggests the assumption that a constraint on applicable heuristics which is sufficiently strong for the delay of a purchase will develop, especially with medium to large assortments. Consequently, the decision cost, especially from medium-sized assortments on, should function as a mediator between variety and purchase probability.

$\mathrm{H} 2$ : with large assortments, the negative effect of variety is mediated by the decision cost.

Proponents of regret theory argue that rejected options play a strong role in consumer choice. For example, consumers who decide to purchase a digital camera find themselves in a situation where the decision in favor of one product necessarily results in the rejection of competing alternatives. If the consumer decides to purchase a cheaper lower quality alternative he rejects a high-end offering; if she opts for a feature rich, expensive camera she rejects cheaper ones. Following regret theory, every rejected option can lead to regret. In this state, individuals ponder the mistake made and suffer under the belief that they could have made a better decision.

Analogous to utility and anticipated utility, the literature distinguishes between two forms of regret: on the one hand, after a decision, regret arises in cases where it has become evident that a rejected option would have been the better choice (experienced regret). On the other hand, individuals anticipate before decisions that they may feel regret over their choice (anticipated regret) (Zeelenberg 1999a). Loomes and Sugden $(1982,820)$ note regarding this issue: “... regret rests on two fundamental assumptions; first people experience the sensation we call regret [...]; and second, that in making decisions [...] they try to anticipate and take account for those sensations...'. In the case where the decision-maker cognitively anticipates regret, anticipated regret leads to regret-as-possibility entering into decision-making. Regret can thus have an influence on the decision without actually being felt.

Decision theorists argue that perceived variety increases anticipated regret (Loewenstein 1999; Iyengar and Lepper 2000). Because of product variety, consumers are confronted not only with effort but also with psychological costs in the form of doubt, uncertainty and mental strain. If customers expect post-purchase anguished thoughts about whether they have made an optimal purchase, they will necessarily see themselves as bearing even more responsibility if the assortment is larger or the freedom of choice is greater (Weiner 1982; Schwartz et al. 2002). While with a small selection, justification on the basis of a lack of options is possible, a large selection allows less room to maneuver psychologically. The consumer finds herself confronted with the psychological pressure of having to accept personal responsibility for potentially negative experiences. Moreover, more alternatives inevitably result in the consumer having to reject more options. While with a range of six digital cameras, five will be rejected, with 30 , the customer is 
forced to decide against 29 others. Consequently, for the decision-maker faced with a larger number of options, it is simply more probable that a sub-optimal choice may be made (Wathieu et al. 2002; Schwartz 2000).

Regret has been shown as one of the major drivers of the decision to postpone a purchase (Beattie et al. 1994; Zeelenberg et al. 1996). A clear and frequently replicated result is that individuals in situations of negative experience feel more regret if the experience is a result of personal action than if the experience is preceded by inaction (Gilovich and Medvec 1995; Kahneman and Tversky 1982; Landman 1987). A shareholder, for example, will experience particularly deep regret if he sells his stock and then later discovers that the stock price has risen substantially. That same lost profit, however, would inspire markedly less regret if the price of his stock had not changed and he had foregone the chance to take a stake in the same positive stock price development but with another company (Kahneman and Tversky 1982). In terms of the monetary gain both cases are identical. However, the second case is psychologically different from the first in that active action would have been needed to make the profit.

Given this background, if individuals want to avoid future regret, they would be well advised to undertake as little active action as possible. In other words, it can be expected that abstaining from imminent action is a direct function of anticipated regret. Indeed, it has been shown that individuals increasingly tend toward inaction if the potential results of their options for action include especially negative eventualities (Baron and Ritov 1994). Individuals are also more apt to postpone a purchase if they are explicitly asked to consider regret, if they feel a higher degree of responsibility for a particular course of action or expect direct feedback regarding the quality of their decision (Kordes-de Vaal 1996; Ritov and Baron 1995). With each of these contexts, anticipated regret increases in lockstep with increased abstention from action. To summarize, it can be stated that anticipation of regret is likely to favor inaction (Kahneman and Tversky 1982).

Analogous to evaluation cost, it cannot be assumed that anticipated regret is able to generally explain the relation between product variety and purchase probability. As pointed out earlier, a number of causes of anticipated regret can be identified. Additional options should be able to have an effect on regret only when consumers see themselves as unable to make a completely well thought-out purchase decision. With smaller assortments, the effect of variety on regret can be seen as ambiguous because with extremely small assortments, the assumed lack of potentially attractive options may also lead to regret (Greenleaf and Lehmann 1995). With large assortments, on the other hand, this phenomenon can be expected, if at all, then only to a minor degree. In this context, the positive relation between the number of alternatives and anticipated regret delineated earlier should clearly dominate.

H3: with large assortments, the negative effect of variety is mediated by anticipated regret.

If a purchase decision leads to anticipated regret, then there should arise two other direct effects on the constructs under discussion here. Proponents of regret theory argue that even rejected options determine the utility of a selected alternative (Tsiros 1998; Tsiros and Mittal 2000). For example, the decision to go to the movies also entails the rejection of the alternative of going to a football game. According to 
regret theory, the rejected option influences the utility of the alternative chosen. The knowledge of having given up something pleasant leads to a decrease in the experienced utility of the alternative selected.

As a consequence, there results the relation that additional attractive options can reduce the expected utility of a preferred option (Schwartz 2000). If the decisionmaker can choose only one alternative, then subjective utility of that alternative will only depend on this option itself. When more options are available, each additional alternative could be perceived as a potentially better choice and consequently lower experienced utility. If consumers indeed anticipate this, the following relation should hold:

$\mathrm{H} 4$ : anticipated regret has a negative influence on the anticipated utility of the preferred alternative.

As explained above, decision-makers can be induced to invest more effort in a purchase decision when they see a utility in additional effort. If individuals experience anticipated regret, the utility of a copious deliberation process lies in the reduction of negative post-purchase effects. It is especially important to consider in such a case that regret can be triggered, not least of all, by a quick or careless decision-making process. For example, a student who fails a test will experience especially deep regret if he has failed to prepare himself thoroughly. On the other hand, if he is unsuccessful in spite of having prepared himself as well as possible, he may simply experience disappointment instead of regret since the result is outside of his sphere of responsibility (van Dijk et al. 1999). Accordingly, individuals who anticipate regret before their purchase should try to reduce the danger of postpurchase regret through intensive deliberation.

For regret, it is essential to be able to reflect upon a potentially better conduct (Zeelenberg et al. 1998). A purchase decision which is not well thought-out is completely in the decision-maker's realm of responsibility. Moreover, individuals find it particularly easy after the purchase to mentally simulate intensive prepurchase efforts (Zeelenberg 1999b). Individuals are evidently willing to invest mental and temporal resources in order not to have to reproach themselves for having committed a mistake which could have been avoided through careful deliberation. Consequently, anticipated regret stands in direct causal relation with elaborate purchase decisions (Zeelenberg 1999a).

H5: anticipated regret has a positive effect on the evaluation costs of the purchase decision.

Figure 1 presents a graphical model that summarizes the focal concepts and relationships considered in this article. Note that the relation between perceived variety and purchase probability, according to theory, does not represent a linear but rather an inverted U-shaped relation. The relation is shown as a broken line since while the mediators are controlled, no significant or at least a weak effect should be present. The three variables anticipated utility, anticipated regret and evaluation costs were hypothesized to explain the relationship between variety and purchase probability. In addition, our discussion suggests that regret drives both anticipated product utility and anticipated regret. According to theory, variety should have a stronger impact on anticipated product utility for small than for large assortments. 


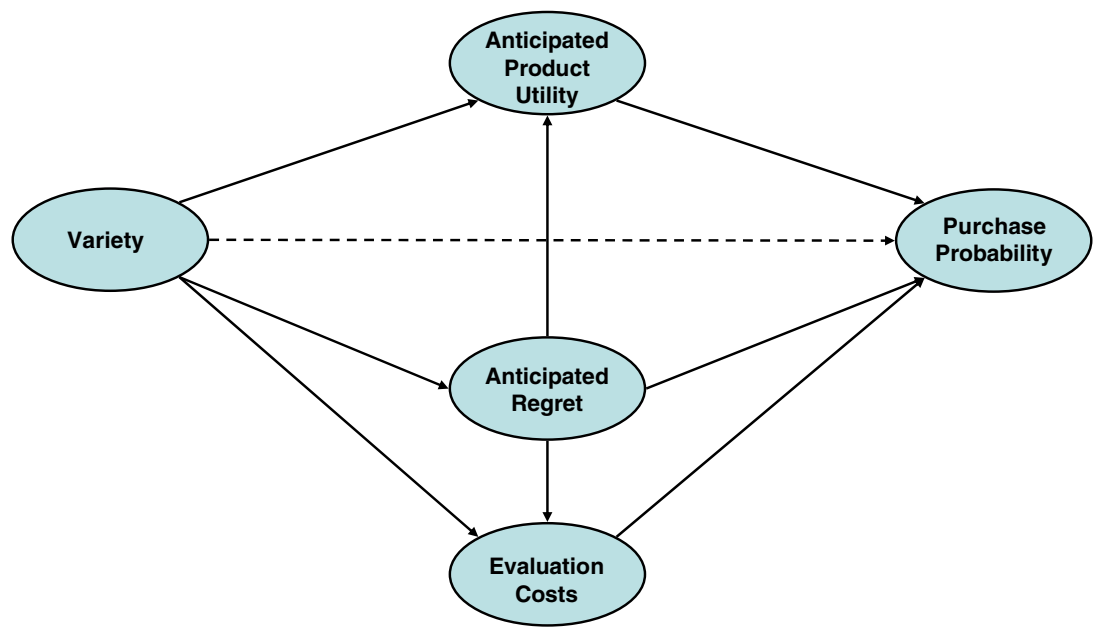

Fig. 1 Research model

Conversely, anticipated regret as well as evaluation costs should be stronger affected by variety in large rather than small assortments.

\section{Empirical analysis}

\subsection{Data and measures}

We utilize data from a probability sample of actual customers of three different stores of a major electronics retailing chain in Germany. Our data is thus related to actual purchase decisions as opposed to hypothetical decisions made in a lab setting. This is important, since key constructs such as anticipated regret may be experienced differently when individuals are faced with decisions with actual consequences rather than hypothetical decisions made in a lab setting (Luce et al. 2001). Participants were contacted during August 2004. The sample was restricted on customers who intended to buy either a digital camera or a DVD player. These two product categories were chosen because they varied in variety across categories and stores.

To insure actual deliberation about the available options only those customers were contacted who deliberated more than $5 \mathrm{~min}$ about the available options. Respondents were further screened by including only those customers who explicitly stated that they indented to purchase a product when entering the store. Customers who met both criteria were personally interviewed using a standardized survey instrument that consisted of measures for each construct of our model. Out of 596 answers obtained, 13 had to be eliminated due to inconsistent answers and a large number of missing values. The remaining 583 responses consisted of 295 
(50.60\%) customers who purchased a product and $288(49.40 \%)$ who decided not to purchase. $367(62.95 \%)$ individuals were interested in a digital camera and 216 $(37.05 \%)$ in a DVD player. Participants' ages ranged from 18 to 75 years and were $28.72 \%$ female with a mean age of 38.31 years.

Whenever possible, we employed extant literature to measure our model's constructs. Anticipated product utility was measured using a four-item scale that was based on Kahn and Wansink (2004). For measuring evaluation costs a five-item scale based on Loewenstein (1999) as well as Huffman and Kahn (1998) was used. The scale for anticipated regret was based on Chatterjee and Heath (1996) as well as Sweeney et al. (2000). All items were measured on seven-point scales. In addition, we asked participants whether or not they purchased a product (yes/no) and about their perception of the numbers of alternatives being offered on a 13-point scale.

We employed LISREL to conduct confirmatory factor analysis of the continuous scales used (omitting the binary purchase variable). The measurement model has high levels of internal consistency, convergent validity, and discriminant validity (Bagozzi 1980). Globally, the measurement model with all 14 continuous indicators achieves a good fit $\left(\chi^{2}=222, d f=72\right.$, RMSEA $=0.06$, CFI $=0.98$, TLI $\left.=0.98\right)$. Coefficient alpha for the three multi-item constructs ranged from 0.86 to 0.90 and average variance extracted from 0.61 to 0.66 . We compared the average variance extracted with the variance shared between all construct pairs to investigate discriminant validity (Fornell and Larcker 1981). Internal consistency exceeds

Table 1 Measurement model of multi item constructs

\begin{tabular}{llll}
\hline Latent and manifest variables & Standardized & Unstandardized & Standard error \\
\hline Anticipated product utility $\left(\rho_{\xi}=0.61, \alpha=0.86\right)^{\mathrm{a}}$ & 0.71 & 0.87 & \\
Need fulfillment & 0.87 & 1.00 & 0.05 \\
Pleasure & 0.77 & 0.88 & - \\
Usefullness & 0.79 & 0.90 & 0.04 \\
Satisfaction & & & 0.04 \\
Anticipated regret $\left(\rho_{\xi}=0.66, \alpha=0.89\right)$ & 0.82 & 1.00 & \\
Potential superiority of competing products & 0.81 & 0.92 & - \\
Certainty to identify best product $(R)^{\mathrm{b}}$ & 0.79 & 0.98 & 0.04 \\
Good feeling about decision $(R)$ & 0.83 & 0.97 & 0.05 \\
Fear irreversibility & & & 0.04 \\
Evaluation costs $\left(\rho_{\xi}=0.65, \alpha=0.90\right)$ & 0.79 & 0.88 & \\
Energy & 0.78 & 0.86 & 0.04 \\
Effort & 0.77 & 0.82 & 0.04 \\
Time & 0.84 & 1.00 & 0.04 \\
Amount of thinking & 0.84 & 0.97 & - \\
Complexity of decision & & & 0.04 \\
\hline
\end{tabular}

\footnotetext{
${ }^{a} \rho_{\xi}$ is Fornell and Larcker's (1981) average variance extracted, a measure of convergent validity. $\alpha$ is Cronbachs coefficient alpha

b $R$ Reversed coded items

All factor loadings are statistically significant at $p<0.01$
} 


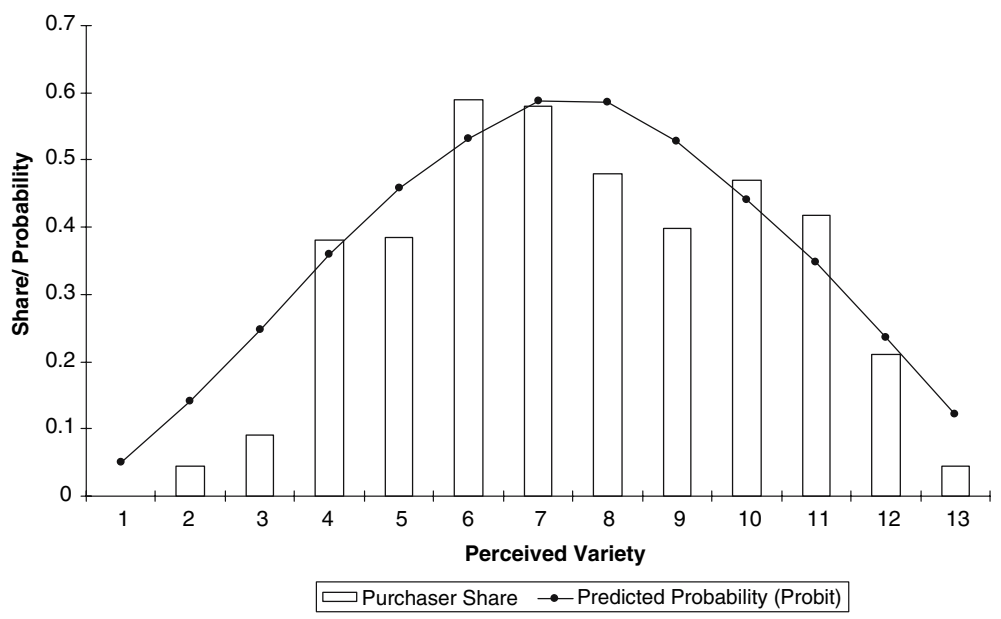

Fig. 2 Relationship between perceived variety and probability to purchase a product

external consistency for each construct (the average difference between the variance extracted and shared variance was 0.38). Details of the measurement model as well as the indicators used are given in Table 1.

\subsection{Model estimation}

Our data is consistent with previous research that had evidenced an inverted Ushaped relation between variety and the probability of purchasing a product. To test this, we model the simple main effect of perceived variety on the latent probit of purchasing a product. ${ }^{1}$ Since we expect a non-linear effect, we employ degree two polynomials of perceived variety (see Aiken and West 1991, 64-67 for a detailed discussion on the estimation of curvilinear relationships). Consistent with prior empirical work, we find a positive effect of variety for the linear term $(\beta=0.65$, $p<0.001)$ and a negative effect for the squared term $(\beta=-0.04, p<0.001)$, suggesting that the positive effect of variety decreases as variety increases. A likelihood-ratio test comparing a linear model without the squared term with the nonlinear model provides evidence that the inclusion of the squared term improves $\log$ likelihood $\left(\chi^{2}(1)=20.44, p<0.001\right)$. To interpret the probit coefficients in more detail, we calculate the predicted probability of purchasing a product based on the probit regression for each of the 13 levels of variety perception (as well as the squared term). A graphical representation of these values as well as the actual share of customers purchasing a product is shown in Fig. 2.

\footnotetext{
${ }^{1}$ We decided to test the inverted U-shaped relation by modeling the data on the individual level. Instead of the probit model, we may have estimated a logit model. To identify probit and logit models the variance of the error term needs to be fixed. In probit an error variance of 1 is assumed, logit models assume a variance of $\pi / 3$. While this assumption is arbitrary, it does not affect the value of predicted probabilities interpreted below (see Long 1997, 49-50 for a mathematical proof).
} 
Figure 2 reveals that the positive effect of variety not only decreases with perceived assortment size but becomes negative for large assortments. Furthermore, it is interesting to note that extremely low and extremely high values of perceived variety lead to different predicted purchase probabilities. Participants who perceived variety as extremely low ( 1 on the 13 -point scale) have a $5.00 \%$ probability of making a purchase. Customers who perceive variety as extremely high (13 on the 13-point scale) have a substantially higher predicted probability of $12.21 \%$. Apparently, very small assortments are more likely to lead customers to postpone purchases than very large assortments. In addition, Fig. 2 shows that there seems to be an optimal medium amount of perceived variety.

To test our hypotheses on the causal mechanism between variety and purchase probability, we test for mediation. Generally, mediation can be conceptualized by a series of regressions

$$
\begin{gathered}
\text { Model 1: } Y=\beta_{1}+\tau X_{\mathrm{E}}+\varepsilon_{1} \\
\text { Model 2: } Y=\beta_{2}+\tau^{6} X_{\mathrm{E}}+\beta X_{\mathrm{M}}+\varepsilon_{2} \\
\text { Model 3: } X_{\mathrm{M}}=\beta_{3}+\alpha X_{\mathrm{E}}+\varepsilon_{3}
\end{gathered}
$$

Baron and Kenny (1986) suggest three conditions for mediation. The first condition tests whether the exogenous variable $\left(X_{\mathrm{E}}\right)$ determines the target variable $(Y)$ if the mediator is not considered (Eq. 1). The second condition entails the test of whether each preceding variable can explain variance in the variable that follows it. Thus, the mediator $\left(X_{\mathrm{M}}\right)$ should have a significant effect on $Y$ (Eq. 2), and $X_{\mathrm{M}}$, in turn, should be determined by $X_{\mathrm{E}}$ (Eq. 3). Finally, $\tau^{\prime}$ should not be significant when controlling for $X_{\mathrm{M}}$. Baron and Kenny (1986) speak in this case of complete mediation but point out that in socio-scientific research, multiple mediators are common, and often only partial mediation $\left(\tau^{\prime}<\tau\right)$ can be proven. The testing of individual causal relationships is concerned less with a statistical test for mediation than with a test for necessary conditions (MacKinnon et al. 2002). The method does not permit the estimation of standard errors or confidence intervals for the mediated effect. Furthermore, it is difficult to extend the procedure to multiple mediators (Lehmann 2001).

A statistical test for the intervening effect must investigate statistical significance of the difference $\tau-\tau^{\prime}$ (Freedman and Schatzkin 1992; MacKinnon et al. 1995; Clogg et al. 1992). If this difference is significant, partial mediation is established. In addition, if $|\tau|>0$ and $\tau^{\prime}=0$, data provides evidence for full mediation. The effect $\tau$ of the exogenous variable on the target variable without controlling for the mediator corresponds to the total effect between both variables, i.e., the sum of the direct and indirect effect while the mediator is controlled $\left(\tau=\tau^{\prime}+\alpha \beta\right)$. The difference of the coefficients $\tau-\tau^{\prime}$ thus corresponds to the product $\alpha \beta$ (MacKinnon and Dwyer 1993; MacKinnon et al. 1995).

Accordingly, mediation can be tested by concentrating on the product of the coefficients or the indirect effect between the exogenous variable and mediator $(\alpha)$ and mediator and target variable $(\beta)$. The most widely applied test of this kind is the test of Sobel (1982) which tests the product of coefficients over its standard error computed by 


$$
\sigma_{\alpha \beta}=\sqrt{\alpha^{2} \sigma_{\beta}^{2}+\beta^{2} \sigma_{\alpha}^{2}}
$$

Simulation studies for mediation tests show especially small Type I and Type II errors for this procedure (MacKinnon et al. 1995). In the following, this test will be applied to the unobserved probit to purchase or not to purchase $\left(Y^{*}\right)$ as a dependent variable. Equations (1) and (2) are then:

$$
\begin{gathered}
\text { Model 1: } Y^{*}=\beta_{1}+\tau X_{\mathrm{E}}+\delta_{1} \\
\text { Model 2: } Y^{*}=\beta_{2}+\tau^{6} X_{\mathrm{E}}+\beta X_{\mathrm{M}}+\delta_{2}
\end{gathered}
$$

In order to define a scale for the unobserved variable $Y^{*}$, the variance of the error term in probit regressions is fixed to 1 . As the error variance is fixed, the values for $\tau^{\prime}$ und $\beta$ are dependent not only on $X_{\mathrm{E}}$ und $X_{\mathrm{M}}$ but also on the remaining explanatory variables. Correspondingly, the difference $\tau^{\prime}-\tau$ cannot be interpreted as it is with a linear regression since here no observable fixed variance of the dependent variables is present. Accordingly, especially high values for $\beta$ (which make mediation more probable) lead to very small values for $\tau^{\prime}-\tau$, i.e., to substantial Type II errors (MacKinnon and Dwyer 1993). MacKinnon and Dwyer (1993) suggest a standardization procedure and show that, with the aid of this procedure, reliable Sobel tests for mediation can be conducted. In the following we will therefore report standardized coefficients and conduct Sobel tests based on this method to investigate the significance of the difference $\tau^{\prime}-\tau$, i.e., whether the hypothesized variables mediate the relation between variety and purchase probability.

Since all mediating variables represent unobserved latent constructs, we employ structural equation modeling to estimate the direct and indirect effects of the model presented in Fig. 1. We had predicted different causal mechanisms for low and high variety assortments. To analyze these differences, we perform a median split $(\leq 7$, $>7$ ) of perceived variety and analyze the small to medium and medium to large variety group of customers separately. We estimate our model in Fig. 1 using Muthén's (1984) continuous/categorical variable methodology based on robust weighted least squares. ${ }^{2}$ Based on the standardized parameter estimates we conducted Sobel-tests to investigate whether the three mediating variables evaluation costs, anticipated regret and anticipated product utility serve to explain the causal mechanism between variety and purchase probability.

\subsection{The positive effect of product variety}

In a first step, we estimated our full model of Fig. 1 for the low to medium variety assortment perception (the increasing part of the slope in Fig. 2). Again, we used the

\footnotetext{
2 The more frequently used maximum likelihood (ML) method for model estimation is not applicable in our case since our depended variable has the two binary values of "purchase" and "no purchase". In such cases ML would result in inflated $\chi^{2}$ fit statistics, biased model parameters and standard errors (Hutchinson and Olmos 1998; Green et al. 1997; Muthén and Kaplan 1992; Babakus et al. 1987). Therefore, we estimate our model based on polychoric correlations instead of variances and covariances (as in ML estimation) (see Flora and Curran 2004 for a more detailed discussion).
} 
probit link function for model estimation. Overall model fit was acceptable $\left(\chi^{2}=257, d f=83\right.$, RMSEA $=0.08, \mathrm{CFI}=0.94$, TLI $\left.=0.92\right)$.

While hypothesis 4 predicted a negative effect of anticipated regret on anticipated product utility, hypothesis 5 predicted a positive effect of anticipated regret on evaluation costs. Both hypotheses are supported at $p<0.001$ (see Table 2). We find a negative effect of regret on utility $(\beta=-0.47)$ and a positive effect of regret on evaluation costs $(\beta=0.62)$. As expected, neither anticipated regret nor evaluation costs are driven by perceived variety in the case of low variety assortments $(p>0.10)$. Interestingly, both constructs are important in explaining purchase probability. Evaluation costs exert a strong direct impact on the probability of making a purchase $(\beta=-0.32, p<0.001)$. The direct effect of anticipated regret is not significant $(p>0.10)$ when controlling for evaluation costs. However, the product of the coefficients for the effect of regret on evaluation costs and evaluation costs on purchase probability is significant at $p<0.001\left(\beta_{\text {indirect }}=-0.19\right)$. This means that the total effect of anticipated regret on purchase probability is indirect (mediated) by evaluation costs.

Obviously, anticipated product utility should have an additional positive impact on purchase likelihood, which is what we find $(\beta=0.19, p<0.05)$. We also find that utility is driven by perceived variety $(\beta=0.30, p<0.001)$. However, we obtain only partial support for Hypothesis 1, which predicted that anticipated product utility would explain the positive relationship between variety and purchase likelihood for small assortments. When we ran a model without all mediators, we found a significant positive effect of variety on purchase probability $(\beta=0.18, p<0.01)$. However, this effect decreased only slightly to $\beta=0.13(p<0.10)$ when we added anticipated utility as a mediator to this model. It further decreased to $\beta=0.11$ $(p=0.10)$ when we estimated the full model with all mediators (see Table 2). A Sobel test of the indirect effect of perceived variety over utility on purchase likelihood is only marginally significant. The indirect effect of $0.05(p<0.10)$ demonstrates that utility explains only 0.05 of the total effect of 0.18 between variety and purchase probability. This corresponds to the difference in coefficients between the model without all mediators and the one including only anticipated utility ( $\tau^{\prime}-\tau$ in Eqs. 5 and 6$)$.

None of the other indirect links between variety and purchase probability is significant $\left(p_{\text {Sobel }}>0.20\right)$, i.e. evaluation costs and anticipated regret also do not serve to explain the relation between variety and purchase probability for low variety assortments. Consequently, we must conclude that additional constructs not measured in this study seem to be necessary in order to fully account for the positive relationship between variety and purchase probability.

\subsection{The negative effect of product variety}

Overall model fit for the medium to high variety group (the decreasing part of the slope in Fig. 2) was better $\left(\chi^{2}=143, d f=83\right.$, RMSEA $=0.05$, CFI $=0.97$, TLI $=0.96)$. Consistent with the low to medium variety group, the results support Hypotheses 5, i.e. anticipated regret increases evaluation costs $(\beta=-0.68$, 
Table 2 Parameter estimates and mediation tests for theoretical model

\begin{tabular}{|c|c|c|c|c|}
\hline \multirow[t]{2}{*}{ Dependent variables with predictors beneath } & \multicolumn{2}{|c|}{$\begin{array}{l}\text { Low to medium } \\
\text { variety }\end{array}$} & \multicolumn{2}{|c|}{$\begin{array}{l}\text { Medium to high } \\
\text { variety }\end{array}$} \\
\hline & $b^{a}$ & $p$ & $\mathrm{~b}^{\mathrm{a}}$ & $p$ \\
\hline \multicolumn{5}{|l|}{ Direct effects } \\
\hline Anticipated product utility & $\left(r^{2}=0.31\right)^{\mathrm{b}}$ & & \multicolumn{2}{|l|}{$\left(r^{2}=0.04\right)$} \\
\hline Perceived variety & 0.30 & 0.00 & -0.10 & 0.16 \\
\hline Anticipated regret & -0.47 & 0.00 & -0.14 & 0.08 \\
\hline Ancitipated regret & $\left(r^{2}=0.00\right)$ & & \multicolumn{2}{|c|}{$\left(r^{2}=0.17\right)$} \\
\hline Perceived variety & -0.02 & 0.77 & 0.41 & 0.00 \\
\hline Evaluation costs & $\left(r^{2}=0.38\right)$ & & \multicolumn{2}{|c|}{$\left(r^{2}=0.52\right)$} \\
\hline Perceived variety & -0.01 & 0.79 & 0.10 & 0.09 \\
\hline Anticipated regret & 0.62 & 0.00 & 0.68 & 0.00 \\
\hline Purchase probability & $\left(r^{2}=0.27\right)$ & & \multicolumn{2}{|c|}{$\left(r^{2}=0.32\right)$} \\
\hline Perceived variety & 0.11 & 0.10 & -0.08 & 0.39 \\
\hline Anticipated product utility & 0.19 & 0.04 & 0.26 & 0.00 \\
\hline Anticipated regret & -0.12 & 0.33 & 0.20 & 0.12 \\
\hline Evaluation costs & -0.32 & 0.00 & -0.56 & 0.00 \\
\hline \multicolumn{5}{|l|}{ Indirect effects (or $\left.\tau-\tau^{\prime}\right)^{\mathrm{c}}$} \\
\hline \multicolumn{5}{|l|}{ Purchase probability } \\
\hline Perceived variety $\rightarrow$ anticipated regret & 0.00 & 0.77 & 0.08 & 0.13 \\
\hline Perceived variety $\rightarrow$ evaluation costs & 0.00 & 0.79 & -0.05 & 0.10 \\
\hline Anticipated regret $\rightarrow$ evaluation costs & -0.19 & 0.00 & -0.38 & 0.00 \\
\hline Perceived variety $\rightarrow$ anticipated regret $\rightarrow$ evaluation costs & 0.00 & 0.77 & -0.16 & 0.00 \\
\hline Perceived variety $\rightarrow$ anticipated product utility & 0.05 & 0.05 & -0.03 & 0.19 \\
\hline Anticipated regret $\rightarrow$ anticipated product utility & -0.09 & 0.05 & -0.04 & 0.15 \\
\hline $\begin{array}{l}\text { Perceived variety } \rightarrow \text { anticipated regret } \rightarrow \text { anticipated product } \\
\text { utility }\end{array}$ & 0.00 & 0.77 & -0.02 & 0.16 \\
\hline \multicolumn{5}{|l|}{ Evaluation costs } \\
\hline Perceived variety $\rightarrow$ anticipated regret & -0.01 & 0.77 & 0.28 & 0.00 \\
\hline \multicolumn{5}{|l|}{ Anticipated product utility } \\
\hline Perceived variety $\rightarrow$ anticipated regret & 0.00 & 0.77 & -0.06 & 0.09 \\
\hline
\end{tabular}

${ }^{a}$ b Represents standardized parameter estimates

b $r^{2}$ Represents variances explained in exogenous constructs by exogenous and endogenous antecedent constructs

${ }^{c} \tau-\tau^{\prime}$ Represents difference in coefficients for the effect of perceived variety without $(\tau)$ and with mediator $\left(\tau^{\prime}\right)$

$p<0.001)$. However, we find only weak evidence for H4. Anticipated regret decreases anticipated product utility by $\beta=-0.19(p<0.10$, see Table 2$)$.

In contrast to the low to medium variety group, we find no direct effect of variety on anticipated product utility $(p>0.10)$. However, we find a small and negative indirect effect of variety over anticipated regret on utility $\left(\beta_{\text {indirect }}=-0.06\right.$, $\left.p_{\text {Sobel }}<0.10\right)$. The effect is counterintuitive since added options should enable 
consumers to find a product that matches their preferences more closely. However, more options also increase potential regret, and the fear of regret in turn decreases expected utility. As variety increases beyond an optimal amount, the negative indirect effect seems to dominate the positive one.

Again, we also ran a simple model without all mediators. As expected, we found a significant negative effect of variety on purchase probability $(\beta=-0.25$, $p<0.01)$. Estimation of the full model, however, resulted in a non-significant relation between both variables $(\beta=-0.08, p>0.10)$. Consistent with Hypothesis 1 and in contrast to the low variety analysis, no evidence could be found for an indirect effect over utility on purchase likelihood $\left(\beta_{\text {indirect }}-0.03, p_{\text {Sobel }}>0.10\right)$. Apparently, anticipated product utility does not serve to explain the negative relationship between variety and purchase likelihood for high variety assortments. Hypotheses 2 and 3 had instead predicted that increased evaluation costs and anticipated regret would each mediate the link for large assortments. However, the negative indirect effect for evaluation costs is weak $\left(\beta_{\text {indirect }}=-0.05, p_{\text {Sobel }}<0.10\right)$ and the indirect effect for anticipated regret is not significant. This means evaluation costs alone cannot fully account for the strong total effect between variety and purchase probability $(\beta=-0.25$ is only diminished by -0.05 due to evaluation costs).

Table 2 reveals the reason for both results. While we find no significant direct effect of anticipated regret on purchase likelihood $(\beta=0.02, p>0.10)$, there is a strong indirect effect over evaluation costs $\left(\beta_{\text {indirect }}=-0.38, \mathrm{p}_{\text {Sobel }}<0.001\right)$. This means that the effect of anticipated regret on purchase probability fully flows through evaluation costs. The impact of perceived variety on evaluation costs, on the other hand, is mostly explained by anticipated regret as evidenced by a weak direct $(\beta=0.10, p<0.10)$ and strong indirect effect $\left(\beta_{\text {indirect }}=0.28, p_{\text {Sobel }}<0.001\right)$.

According to these results, the relationship is more complex than initially hypothesized. Apparently, the bulk of the negative link between variety and purchase probability is explained by anticipated regret and evaluation costs in conjunction. Variety seems to have a strong negative effect only if it increases anticipated regret and only if anticipated regret in turn increases evaluation costs, which then decrease purchase probability. This is supported by a significant indirect effect over both constructs $\left(\beta_{\text {indirect }}=-0.16, p_{\text {Sobel }}<0.001\right)$.

In summary, the results for the medium to high variety group differ markedly from the low to medium variety group. While the positive effect of variety is partially mediated by anticipated utility, the negative effect of variety is fully mediated by two different streams. First, there is a weak indirect effect over evaluation costs. Second, there is a strong indirect effect of variety on anticipated regret, which in turn impacts evaluation costs, which then drive purchase probability. Across both groups evaluation costs directly reduce purchase probability, and anticipated product utility directly increases purchase probability. Interestingly, both analyses show that anticipated regret has a negative but only indirect effect over anticipated product utility (in the case of low variety) and via evaluation costs (in the case of high variety) on the likelihood of making a purchase. 


\section{Discussion and implications}

This paper makes several contributions. First, it provides additional evidence for the positive but also negative impact of variety on consumer's interest in purchasing a product. Consistent with previous empirical results, it has been shown that variety increases purchase probability only up to an optimal point. After that, variety demotivates consumers from making a purchase. Second, it was demonstrated that the negative effect of variety operates via anticipated regret and evaluation costs and that the positive effect of variety operates via anticipated utility. This paper has provided evidence that anticipated regret, presumably one of the most potent factors in decision making (Schwartz et al. 2002), operates only indirectly on purchase likelihood by decreasing anticipated utility in small assortments and by increasing evaluation costs in large assortments. In particular, it seems that anticipated regret only exerts its negative effect when it serves to increase evaluation costs (see also Heitmann and Herrmann 2006).

These findings have several implications for retailers as well as manufacturers managing assortments. First and foremost, it shows the importance of limiting variety. Manufacturers but also retailers face high, oftentimes hidden costs from large variety assortments such as reduced capabilities for research and development or increased complexity in quality control. All of these costs are difficult to quantify and may have only long-term effects through increased customer complaints, necessary revisions or even withdrawals of products. As this paper shows, additional costs are not only incurred by the companies providing high variety assortments but also by the customers who are faced with the task of identifying the one best product for their preferences. This task has been shown to reduce purchase likelihood in the case of high variety assortments. As a consequence, high variety may, in the worst case, lead companies towards increased costs and decreased revenues.

We can therefore conclude that variety management seems crucial. While retailers may directly influence the total number of product alternatives available to the consumer. Manufacturers may decide about appropriate distribution channels for their products based on the number of alternatives different channels offer. In addition, manufacturers may need to control the variety of their own assortments. Research has shown that customers who encounter a feeling of indecision tend not only to postpone purchases but also to switch to competing assortments (Gourville and Soman 2005). To provide a sense of proportion, when Procter and Gamble reduced their assortment of Head and Shoulders shampoos from 26 to 15, they experienced an increase in sales of $10 \%$ (Schwartz 2000). While such changes in sales can never be attributed to a single factor, this research has provided an additional indication that the assortment reduction may have been one of the drivers.

Despite this, there is evidence that a temporary provision of additional options may be useful to increase visibility for consumers, make use of previous brand investments or to drive competitors out of the market (Kahn 1998). Companies that use such tactics may wish to manage the potential negative effects on demand. This paper has shown that customer's evaluation costs play a central role in this context. One approach consistent with this result would be to try to keep evaluation costs constant as variety increases. This may be done by reducing the number of 
trade-offs. For example, shampoos that differ in terms of amount, scent and price, will be more difficult to compare than alternatives that differ in only one dimension, e.g. scent (Shugan 1980). In addition, retailers may choose to present similar products together while highlighting common and differing product attributes, which has been shown to reduce consumers' evaluation costs and consequently impact purchase decisions (Chernev 1997).

Furthermore, this research has shown that anticipated regret is an important intervening variable between variety and evaluation costs. There are a number of ways in which anticipated regret may be managed. Among others, regret can only arise when individuals are responsible for their own decisions and when decisions seem irreversible (Zeelenberg 1999a). Whenever consumers can avoid making an active choice in the face of potentially suboptimal decisions, anticipated regret is reduced. Companies may decide to feature or recommend one product out of an assortment. Similarly, they may highlight which alternative has been purchased most often by other consumers. Such recommended or frequently purchased products enable consumers to reduce their own perceived responsibility by relying on the judgment of others. In addition, money back guarantees reduce the impression of irreversible decisions and thereby anticipated regret. Although such guarantees are seldom redeemed, they do serve to reduce the fear of irreversible errors in the mind of the consumer.

There are, of course, limitations to this research. One of the main limitations is the data. While useful and derived from "real" consumers, it is limited to consumer electronics, specifically DVD players and digital cameras. The process may differ in other categories that vary in expenditure level, hedonic versus utilitarian aspects, involvement, etc. In addition, customers were asked about their previous decisionmaking process immediately after they had passed through check-out. Despite the small time interval between decision and survey, the data is still based on a recall method that is subject to hindsight bias. Furthermore, a subset of subjects may have had an idea of the variety offered prior to visiting a store. Consequently, we may have omitted customers from our analysis who did not show an interest in the stores we surveyed because they expected variety to be either too low or too high. Thus, the process discovered here may differ depending on the level of prior knowledge about the variety being offered.

It is worth noting that we found only weak evidence for the common assumption made by economists that variety provides a potentially better match between consumers' preferences and the alternatives offered. Expected product utility could explain the causality between variety and purchase probability only in the case of low variety assortments and here only to a limited degree. Nevertheless, variety did increase purchase probability. The question of why this relationship exists is worth investigating in future research. Currently, we can only speculate about further mediating constructs. Potential candidates include stimulation by the perception of freedom of choice (Brehm 1972; Deci and Ryan 1985) or anticipation of future variety seeking (Kahn 1998).

For variety to exert an effect on purchase probability it must be perceived as either large or small. This research on the causal mechanism between variety and purchase probability has consequently concentrated on perceived variety instead of 
the objective number of options. However, the causal mechanisms reported here may also be triggered by additional factors other than actual variety. Factors such as the shelf space utilized (Broniarczyk et al. 1998), the degree of diversity among options (Lehmann 1998) or the number of options matching consumer's screening criteria (Kahn 1998) have also been proposed to impact variety perceptions. In addition, we have questioned customers on the number of options not distinguishing between the variety of products within and across brands. Consistent with the previous argument, the diversity of options and consequently perceived variety may be lower for assortments with few brands and many options per brand than for assortments with many brands and few options per brand. Further research is needed to examine the relationship between perceived and actual variety in more detail. This would be valuable since in certain cases even objectively small assortments may be perceived large enough to demotivate consumers from buying. For example, Chernev (2003) provides results according to which consumers without a preferred attribute combination in mind are demotivated by smaller assortments than consumers with a precise idea of an ideal product.

Several additional directions for future research seem obvious. We were only able to measure whether or not participants bought a product on a single shopping occasion. Participants may have simply delayed the transaction and decided to purchase at a later point in time. Therefore, it would be interesting to replicate our study with a longitudinal design. In addition, further research may concentrate on the customers who decided to buy a product to study the effect on customer retention. Recent research by Heitmann et al. (2007) suggests that the variables captured in this model may have an effect on satisfaction with the product as well as repurchase intent. Other research suggests that those customers who do buy may prefer high variety assortments in the long run, as they enable variety seeking (Bawa 1990). Consequently, research on the long-term effect of high variety assortments on customer retention would be valuable.

\section{References}

Aiken LS, West SG (1991) Multiple regression testing and interpreting interactions. Sage, Newbury Park (CA)

Anderson CJ (2003) The psychology of doing nothing: forms of decision avoidance result from reason and emotion. Psychol Bull 129:139-167

Babakus E, Ferguson CE, Jöreskog KG (1987) The sensitivity of confirmatory maximum likelihood factor analysis to violations of measurement scale and distributional assumptions. J Mark Res 37:72-141

Bagozzi RP (1980) Causal models in marketing. Wiley, New York

Baron RM, Kenny DA (1986) The Moderator-mediator variable distinction in social psychological research. J Pers Soc Psychol 51:1173-1182

Baron J, Ritov I (1994) Reference points and omission bias. Organ Behav Hum Decis Process 59:475498

Bawa K (1990) Modeling inertia and variety seeking tendencies in brand choice behavior. Mark Sci 9:263-278

Beach LR, Mitchell TR (1978) A contingency model for the selection of decision strategies. Acad Manage Rev 3:439-449

Beattie J, Baron J, Hershey JC (1994) Psychological determinants of decision attitude. J Behav Decis Mak 7:129-144 
Bettman JR, Luce MF, Payne JW (1998) Constructive consumer choice processes. J Consum Res 25:187217

Boatwright P, Nunes JC (2001) Reducing assortment: an attribute-based approach. J Mark 65:50-65

Brehm JW (1972) Responses to the loss of freedom: a theory of psychological reactance. General Learning Press, Morristown

Broniarczyk SM, Hoyer WD, McAlister L (1998) Consumers' perceptions of the assortment offered in a grocery category: the impact of item reduction. J Mark Res 35:166-176

Chatterjee S, Heath TB (1996) Conflict and loss aversion in multiattribute choice: the effects of trade-off size and reference dependence on decision difficulty. Organ Behav Hum Decis Process 67:144-155

Chernev A (1997) The effect of common features on brand choice: moderating role of attribute importance. J Consum Res 23:304-310

Chernev A (2003) When more is less and less is more: the role of ideal point availability and assortment in consumer choice. J Consum Res 30:170-183

Chernev A (2006) Decision focus and consumer choice among assortments. J Consum Res 33:50-59

Clogg CC, Petkova E, Shihadeh ES (1992) Statistical methods for analyzing collapsibility in regression models. J Edu Stat 17:51-74

Corbin RM (1980) Decisions that might not get made. In: Wallsten TS (ed) Cognitive processes in choice and decision behavior. Erlbaum, Hilldale pp 47-67

Deci EL, Ryan RM (1985) Intrinsic motivation and self-determination in human behavior. Plenum Press, New York

Dhar R (1997) Consumer preference for a no-choice option. J Consum Res 24:215-231

Flora DB, Curran PJ (2004) An empirical evaluation of alternative methods of estimation for confirmatory factor analysis with ordinal data. Psychol Meth 9:466-491

Ford JK, Schmitt N, Schlechtman SL, Hults BM, Doherty ML (1989) Process tracing methods: contributions, problems, and neglected research questions. Organ Behav Hum Decis Process 43:75117

Fornell C, Larcker DF (1981) Evaluating structural equation models with unobservable variables and measurement error. J Mark Res 18:39-50

Freedman LS, Schatzkin A (1992) Sample size for studying intermediate endpoints with intervention trials of observational studies. Am J Epidemiol 136:1148-1159

Frisch D, Clemen RT (1994) Beyond expected utility: rethinking behavioral decision research. Psychol Bull 116:46-54

Gilovich T, Medvec VH (1995) The experience of regret: what, when and why. Psychol Rev 102:379-395

Glazer R, Kahn BE, Moore WL (1991) The influence of external constraints on brand choice: the lonealternative effect. J Consum Res 18:119-127

Gourville JT, Soman D (2005) Overchoice and assortment type: when and why variety backfires. Mark Sci 24:382-395

Green SB, Akey TM, Flemin KK, Hershberger SL, Marquis M (1997) Effect of the number of scale points on chi-square fit indices in confirmatory factor analysis. Struct Equ Model 4:108-120

Greenleaf EA, Lehmann DR (1995) Reasons for substantial delay in consumer decision making. J Consum Res 22:186-199

Harsanyi JC (1997) Utilities, preferences, and substantive goods. Soc Choice Welfare 12:129-145

Heitmann M, Herrmann A (2006) Providing more or providing less? Accounting for cultural differences in consumers' preference for variety. Int Mark Rev 23:7-24

Heitmann M, Lehmann DR, Herrmann A (2007) Choice goal attainment and decision and consumption satisfaction. J Mark Res 44:234-250

Hicks JR (1939) Value and capital: an inquiry into some fundamental principles of economic theory. Clarendon Press, Oxford

Hutchinson SR, Olmos A (1998) Behavior of descriptive fit indexes in cofirmatory factor analysis using ordered categorical data. Struct Equ Model 5:344-364

Iyengar SS, Lepper MR (2000) When choice is demotivating: can one desire too much of a good thing? J Personal Soc Psychol 79:995-1006

Jacoby J, Speller DE, Kohn CA (1974) Brand choice behavior as a function of information load. J Mark Res 11:63-69

Johnson EJ, Meyer RJ (1984) Compensatory choice models of noncompensatory processes: the effect of varying context. J Consum Res 11:528-541

Kahn BE (1998) Dynamic relationships with customers: high variety strategies. J Acad Mark Sci 26:4553 
Kahn BE, Lehmann DR (1991) Modeling choice among assortments. J Retail 67:274-289

Kahn BE, Wansink B (2004) The influence of assortment structure on perceived variety and consumption quantities. J Consum Res 30:519-533

Kahn BE, More WL, Glazer R (1987) Experiments in constrained choice. J Consum Res 14:96-113

Kahneman D, Tversky A (1982) The psychology of preferences. Sci Am 246:160-173

Kahneman D, Wakker PP, Sarin R (1997) Back to Bentham? Explorations of experienced utility. Q J Econ 112:375-406

Kordes-de Vaal J (1996) Intention and the omission bias: omissions perceived as nondecisions. Acta Psychol 93:161-172

Kreps DM (1979) A representation theorem of preference for flexibility. Econometrica 47:565-577

Lancaster K (1990) The economics of product variety: a survey. Mark Sci 9:189-206

Landman J (1987) Regret and elation following action and inaction: affective responses to positive versus negative outcomes. Personal Soc Psychol Bull 13:524-536

Lehmann DR (1998) Customer reactions to variety: too much of a good thing? J Acad Mark Sci 26:62-65

Lehmann DR (2001) Mediators and moderators. J Consum Psychol 10:89-91

Levav J, Heitmann M, Herrmann A, Iyengar SS (2006) The effect of attribute order and variety on choice demotivation: a field experiment on German car buyers. Adv Consum Res (in press)

Loewenstein G (1999) Is more choice always better? Soc Security Brief: National Academy of Social Insurance 7:7-19

Long JS (1997) Regression models for categorial and limited dependent variables. Thousand Oaks

Loomes G, Sugden R (1982) Regret theory: an alternative theory of rational choice under uncertainty. Econ J 92:805-824

Luce MF, Bettman JR, Payne JW (2001) Emotional decisions: Tradeoff difficulty and coping in consumer choice. Chicago

MacKinnon DP, Dwyer JH (1993) Estimated mediated effects in prevention studies. Eval Rev 17:144-158

MacKinnon DP, Warsi G, Dwyer JH (1995) A simulation study of mediated effect measures. Multivariate Behav Res 30:41-61

MacKinnon DP, Lockwood CM, Hoffman JM, West SG, Sheets V (2002) A comparison of methods to test mediation and other intervening variable effects. Psychol Meth 7:83-104

Malhotra NK (1982) Information load and consumer decision making. J Consum Res 8:419-430

March JG (1978) Bounded rationality, ambiguity, and the engineering of choice. Bell J Econ 9:587-608

Miller GA (1956) The magic number seven plus or minus two: some limits in our capacity for processing information. Psychol Rev 63:81-97

Muthén B (1984) A general structural equation model with dichotomous, ordered categorical, and continuous latent variable indicators. Psychometrika 49:115-132

Muthén B, Kaplan D (1992) A comparison of some methodologies for the factor-analysis of non-normal likert variables: a note on the size of the model. Br J Math Stat Psychol 45:19-30

Payne JW, Bettman JR, Johnson EJ (1993) The adaptive decision maker. Cambridge

Ratchford BT (1982) Cost-benefit models of explaining consumer choice and information seeking behavior. Manage Sci 28:197-211

Ratner RK, Kahn BE, Kahneman D (1999) Choosing less-preferred experiences for the sake of variety. J Consum Research 26:1-15

Ritov I, Baron J (1995) Outcome knowledge, regret and omission bias. Organ Behav Hum Decis Process 64:119-127

Schwartz B (2000) Self-determination: the tyranny of freedom. Am Psychol 55:79-88

Schwartz B, Ward A, Monterosso J, Lyubomirsky S, White K, Lehman DR (2002) Maximizing versus satisficing: happiness is a matter of choice. J Personal Social Psychol 83:1178-1197

Shugan SM (1980) The cost of thinking. J Consum Res 7:99-111

Simon HA (1955) A behavioral model of rational choice. Q J Econ 69:99-118

Simon HA (1990) Invariants of human behavior. Annu Rev Psychol 41:1-19

Sobel ME (1982) Asymptotic confidence intervals for indirect effects in structural equation models. In: Leinhardt S (ed) Sociological methodology. Jossey-Bass, Washington (DC), pp 290-312

Stigler GJ (1961) The economics of information. J Polit Econ 3:213-225

Sweeney JC, Hausknecht D, Soutar GN (2000) Cognitive Dissonance after purchase: a multidimensional scale. Psychol Mark 17:369-385

Timmermans D (1993) The impact of task complexity on information use in multi-attribute decision making. J Behav Decis Mak 6:95-111 
Tsiros M (1998) Effect of regret on post-choice valuation: the case of more than two alternatives. Organ Behav Hum Decis Process 76:48-69

Tsiros M, Mittal V (2000) Regret: a model of its antecedents and consequences in consumer decision making. J Consum Res 26:401-417

Tversky A, Shafir E (1992) Choice under conflict: the dynamics of deferred decision. Psychol Sci 3:358361

van Dijk WW, van der Pligt J, Zeelenberg M (1999) Effort invested in vain: the impact of effort on the intensity of disappointment and regret. Motiv Emot 23:203-220

Vodopivec B (1992) A need theory perspective on the parrlelism of attitude and utility. J Econ Psychol 13:19-37

Wathieu L, Brenner L, Carmon Z, Chattopadhyay A, Drolet A, Gourville JT, Novemsky N, Ratner RK, Wertenbroch K, Wu G (2002) Consumer control and empowerment: a primer. Mark Lett 13:297305

Weiner B (1982) The emotional consequences of causal attribution, In: Clark MS, Fiske ST (eds) Affect and cognition. Erlbaum, Hillsdale (NJ), pp 185-209

Zeelenberg M (1999a) Anticipated regret, expected feedback and behavioral decision making. J Behav Decis Mak 12:93-106

Zeelenberg M (1999b) The use of crying over spilled milk: a note on the rationality and functionality of regret. Philos Psychol 12:325-340

Zeelenberg M, Beattie J, van der Pligt J, de Vries NK (1996) Consequences of regret aversion: effects of expected feedback on risky decision making. Organ Behav Hum Decis Process 65:148-158

Zeelenberg M, van Dijk WW, Manstead ASR (1998) Reconsidering the relation between regret and responsibility. Organ Behav Hum Decis Process 74:254-272

Zipf GK (1949) Human behavior and the principle of least effort. Cambridge 